\title{
Comparison between 3D-reconstruction optical methods applied to bulge-tests through a feed-forward neural network
}

\author{
Damiano Alizzio', Marco Bonfanti ${ }^{2}$, Guido Garozzo ${ }^{3}$, Fabio Lo Savio ${ }^{4}$, Roberto Montanini ${ }^{1}$, \\ Antonino Quattrocchi ${ }^{1}$ \\ ${ }^{1}$ Dept. of Engineering, University of Messina, Cont.da Di Dio, 98166 Messina, Italy \\ 2 Dept. of Electric, Electronic, Informatics Engineering, Univ. of Catania, Via S. Sofia, 54, 95100 Catania, Italy \\ ${ }^{3}$ Zerodivision Systems S.r.I. Piazza S. Francesco n. 1, 56127 Pisa, Italy \\ ${ }^{4}$ Dept. of Civil Engineering and Architecture, University of Catania, Via S. Sofia, 54, 95100 Catania, Italy
}

\section{ABSTRACT}

The mechanical behaviour of rubber-like materials can be investigated through numerous techniques that differ from each other in costs, execution times and parameters described. Bulge test method proved helpful for hyperelastic membranes under plane and equibiaxial stress state. In the present study, bulge tests in force control were carried out on SBR $20 \%$ CB-filled specimens. 3D reconstructions of the dome were achieved through two different stereoscopic techniques, the epipolar geometry and the Digital Image Correlation. Through a Feed-Forward Neural Network (FFNN), these reconstructions were compared with the measurements by a laser triangulation sensor taken as reference. 3D-DIC reconstruction was found to be more accurate. Indeed, bias errors of the 3D-DIC and epipolar techniques with respect to the relative reference values, under creep condition, were $0.53 \mathrm{~mm}$ and $0.87 \mathrm{~mm}$, respectively.

\section{Section: RESEARCH PAPER}

Keywords: Bulge-test; creep; 3D-DIC; epipolar geometry; neural network

Citation: Damiano Alizzio, Marco Bonfanti, Guido Garozzo, Fabio Lo Savio, Roberto Montanini, Antonino Quattrocchi, Comparison between 3Dreconstruction optical methods applied to bulge-tests through a feed-forward neural network, Acta IMEKO, vol. 10, no. 4, article 30, December 2021, identifier: IMEKO-ACTA-10 (2021)-04-30

Section Editor: Roberto Montanini, Università di Messina and Alfredo Cigada, Politecnico di Milano, Italy

Received August 2, 2021; In final form December 4, 2021; Published December 2021

Copyright: This is an open-access article distributed under the terms of the Creative Commons Attribution 3.0 License, which permits unrestricted use, distribution, and reproduction in any medium, provided the original author and source are credited.

Corresponding author: Fabio Lo Savio, e-mail: flosavio@diim.unict.it

\section{INTRODUCTION}

The mechanical characterization of elastomers needs the knowledge of numerous hyperelastic constants describing their highly non-linear mechanical behaviour [1]-[6]. Nowadays a lot of different techniques are used to determine the stress-strain curve, from the most traditional uniaxial tensile-compressive tests, through indentation and equibiaxial tests, up to the bulge tests. Among these, the bulge test is a consolidated technique for the investigation of membranes subjected to an equibiaxial tension state [7], [8]. Such method allows avoiding those edge damages commonly occurring when the specimen is stressed during other types of tests [9].

In bulge tests, a material thin sheet of uniform thickness is clamped between two circular flanges with cavities in the centre to cause, through the insufflation of fluid inside the test chamber, the sheet to deform plastically assuming a semi-spherical shape. Since in isotropic materials the stress state is equibiaxial, the relation between the pressure-displacement and stress-strain curves is unique on the whole dome [10]. In light of the above, this technique, originally used for metallic materials, can be extended also to rubber-like materials, which show typically isotropic behaviour in absence of any previous calendering process which could generate a light transversally isotropy along calendering direction [11], [12].

Under inflation, pressure and displacements are the testing parameters to be constantly monitored, these being the parameters necessary to determine the stress-strain curve.

To achieve a faithful reconstruction of the dome, epipolar geometry [13]-[15] and 3D-Digital Image Correlation (DIC) [16][18] techniques were implemented.

Some of the authors have previously published a device with a mobile crosshead in order to subject an elastomeric membrane to the bulge test in force control (creep) [14], [19].

In the first of the two cited works [14], authors highlighted the $3 \mathrm{D}$ reconstructive technique of a hyperelastic specimen subjected to bulge-test, based on the epipolar geometry. In the 
second one [19], authors trained a Feed-Forward Neural Network (FFNN) on the data acquired using the previous technique, returning a predictive model that provides as output the dome apex height formed by the insufflated specimen.

In the present paper, 5 specimens were bulge-tested in creep condition and, differently from previous works, a comparison between the stereoscopic reconstruction of the specimen dome based on epipolar geometry and 3D-DIC was carried out. This comparison was preferred to be performed through a FFNN independently trained on the base of results from both the reconstructive techniques.

The values of the dome apex height provided by the two FFNN models were, then, compared with the value revealed by using a laser triangulation measurement technique as reference.

\section{THEORETICAL BACKGROUND}

In the bulge test technique, some restrictive assumptions are required to be applied both on the tested material, such as isotropy and incompressibility, and on the geometry of the inflated sample, such as hemispherical shape and small thickness compared to the curvature radius. Under these assumptions, the stress state can be thought as equibiaxial and plane and, according to the Boyle-Mariotte law (fit for thin-walled tanks), is defined by:

$$
\sigma_{c}=\sigma_{a}=\frac{p \cdot R}{2 s},
$$

where $\sigma_{c}$ and $\sigma_{a}$ are, respectively, the circumferential and axial stress, $p$ is the working inflation pressure, $R$ is the curvature radius of the dome and $s$ is the thickness at the dome apex.

At the dome apex each meridian represents a main direction and, then, on its surface all the main strains are equal to each other $\left(\varepsilon_{1}=\varepsilon_{2}=\varepsilon_{e q}\right)$. From the knowledge of the undeformed length $l_{0}$ and the deformed length $l_{d}$ of a membrane finite element, these strains are given by:

$$
\varepsilon_{1}=\varepsilon_{2}=\ln \left(l_{d} / l_{0}\right) \text {. }
$$

From the assumption of incompressibility of the material and in agreement with the well-known Von Mises equation [13]:

$$
\varepsilon_{3}=\ln \left(s / s_{0}\right) \text {, }
$$

where $s_{0}$ is the undeformed specimen thickness.

\section{3D-RECONSTRUCTION METHODS USED}

The stereoscopic technique based on epipolar geometry is a 3D-reconstruction method allows determining the whole dome by identifying, via two cameras, the shifting of a grid printed on the surface of the sample as the dome inflates under creep bulge testing [20]. Acquired images by two cameras are processed through apposite geometrical algorithms and different filters.

The stereoscopic technique based on 3D-DIC is an optical method adopted to measure full-field displacements and strains by applying the cross-correlation to measure shifts in digital images [21]. This method is effective at mapping displacements and deformations thanks to the contrast, necessary to correlate images, generated by the application of an airbrushed speckle pattern on the surface of the sample. The multi-camera system uses a single global axis system. A dedicate software, developed in Matlab ${ }^{\circledR}$ environment, integrates a 2D-DIC subset-based software with different camera calibration algorithms to reconstruct $3 \mathrm{D}$ surfaces from several acquisitions of stereo image pairs. Furthermore, this software contains algorithms for computing and visualizing 3D displacements and strains.

\section{EXPERIMENTAL SETUP}

Bulge tests in force control were performed by a home-made experimental setup already published in previous works of some of the authors [14], [19], [22]. A pneumatic circuit inflates a thin sample clamped between two flanges with adjustable flow rate (Figure 1d). The device is equipped with a sliding crossbar, whose movements are proportional to the membrane inflation. Creep phenomenon was obtained by keeping the pressure constant within the bulge chamber by means of a pressure regulator ( 0.1 bar resolution). In the creep test, after a transient inflation lasting $1 \mathrm{~s}$, the sample was subjected to a constant pressure of 0.55 bar long enough for achieving a complete relaxation, ensuring at the same time that the strain fall within the linear and elastic field.

The core of the stereoscopic acquisition setup (as shown in Figure 1a) is a sliding crossbar, employed to translate two fixedfocus cameras (Imaging Source DMK23G445 monochromatic, equipped with FujiFilm HF35HA-1B lens, having a frame rate up to $30 \mathrm{fps}$ ) in upward/downward direction so to follow the dome apex displacement due to the inflation and, thus, detect the equi-biaxial strain of the specimen. In this way, the dimensions of the captured images depend exclusively on the inflation of the dome and not on its approaching to the camera lenses. The crossbar shifting is made possible by converting the rotary motion of a stepper motor to a vertical translation of the crossbar through a driven shaft and a timing belt (Figure 1b). The linear motion is controlled by an optical system rigidly connected to the crossbar and consisting of a laser diode (working as emitter) and a photodetector (Hamamatsu Si S5973-01 photodiode) placed laterally to the dome (Figure 1c). When the laser beam is interrupted by the inflated dome, the system starts the crossbar shifting via a signal sent to a double $\mathrm{H}$-bridge circuit. The shifting will be stopped once that the photodetector will be newly hit by the laser beam.

The crossbar shifting takes place in steps of $0.125 \mathrm{~mm}$, committing a focal length error of $0.03 \%$ and a negligible focusing error. System accuracy matches the size of the captured pixels $(37.7 \mu \mathrm{m})$. Since the photodiode diameter is greater than that of the laser spot ( $2 \mathrm{~mm}$ vs $1 \mathrm{~mm})$, the laser beam does not affect the spatial resolution of the vertical motion $(0.2 \mathrm{~mm})$.

A laser triangulation sensor (OptoNCDT 1302-200, having a resolution of $0.2 \% \mathrm{FSO}$ ) was placed on the top of the device frame (Figure 1a) and a target-lamina was fixed to the crossbar. These additions were made with the dual purpose of evaluating the uncertainty of the crossbar vertical shifting and indirectly obtaining the measurement of the height of the dome (as shown in Figure 2) to be taken as reference value for both epipolar geometry and 3D-DIC techniques. In other terms, the bias errors of the stereoscopic reconstructions will be calculated by comparing the laser measurement with the measurements from epipolar geometry and 3D-DIC apparata. Particular carefulness was payed for synchronizing, in pseudo real-time, the acquired images to data from pressure transducer and displacement sensor. For this purpose, a NI PXIe-1073 chassis containing acquisition boards (PXIe-6341 and PXI-8252), coupled to a PC in NI LabVIEW ${ }^{\circledR}$ environment, was used. This software also controls the compressed air supply and regulation system. An additional software (Camera Calibration - MATLAB ${ }^{\circledR}$ Toolbox), 

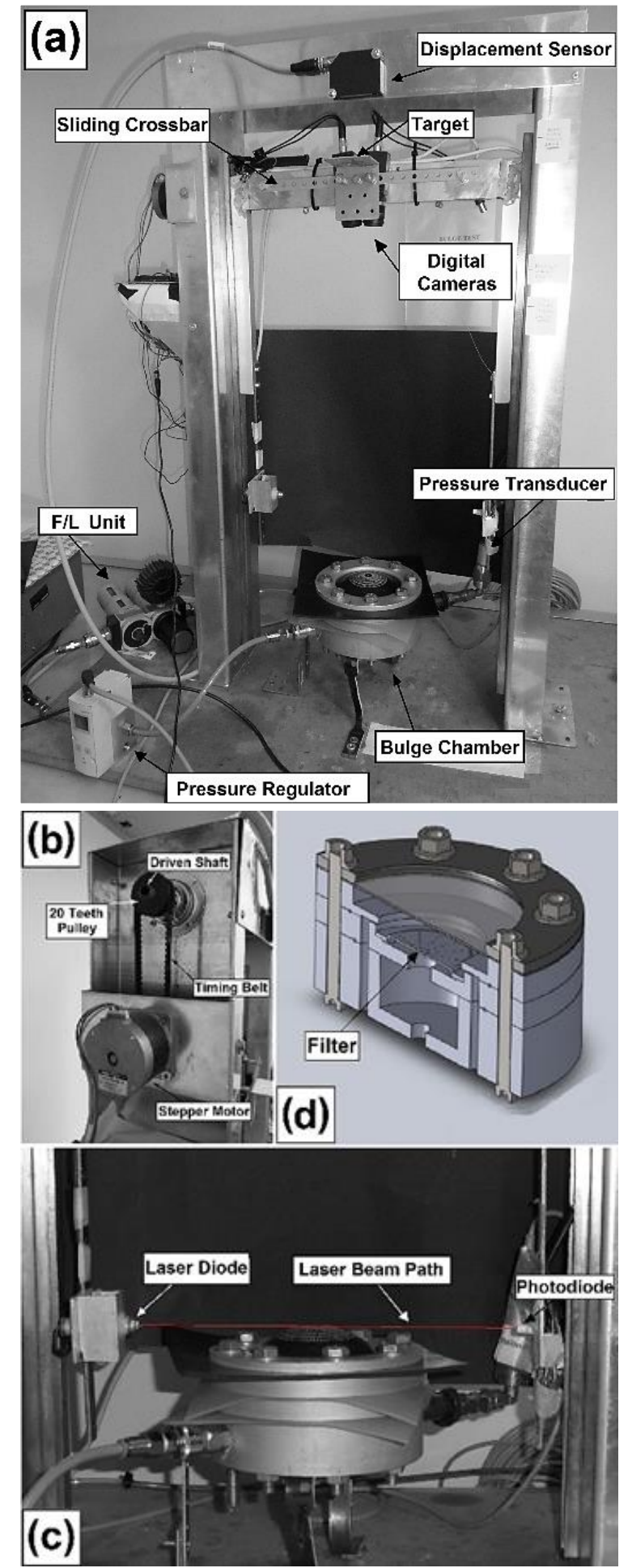

Figure 1. (a) Bulge test setup for epipolar geometry technique; (b) timing belt system; (c) optical system to regulate the vertical shifting; (d) CAD of the bulge chamber cross-section (Courtesy of authors [24]).

using 3D calibration target on the pinhole model [23], was adopted to stereoscopically calibrate the cameras.

To achieve the 3D reconstruction of the dome based on DIC technique, an appropriate GOM ARAMIS 2M LT optical system (Figure 3), also consisting of a double camera but mounted on a tripod, replaced the device with cameras/sliding crossbar used for the epipolar geometry acquisition. In this case, attention was taken during operations of calibration and focusing of cameras.

\section{TEST SAMPLES}

The material tested in this paper was SBR 20\% carbon blackfilled, an artificial elastomer widely used for several applications: from tires, through seals, up to shoe soles [24]-[26]. A set of 5 square specimens $\left(180 \times 180 \mathrm{~mm}^{2}\right)$ were cut from a single $3 \mathrm{~mm}$ thick sheet of elastomer. Each specimen was used for both stereoscopic techniques and, thus, presented two different patterns, one on each side, as shown in Figure 4. On the first side (Figure 4a), adopted for the epipolar reconstruction, a grid consisting of five concentric circles (hence named parallels), a small central circle whose centre corresponded to the top of the dome, and 73 equidistant rays (hence named meridians) radiating from the centre were silk-screened. On the second side (Figure $4 \mathrm{~b})$, useful to carry out the 3D-DIC reconstruction, a pattern of random spots of white paint was airbrushed (with nozzle diameter $\phi=0.18 \mathrm{~mm}$ ).

In this way, the mean value of the diameter of the spots was found to be $\phi=0.23 \mathrm{~mm}$ and their relative surface equals to $0.042 \mathrm{~mm}^{2}$.

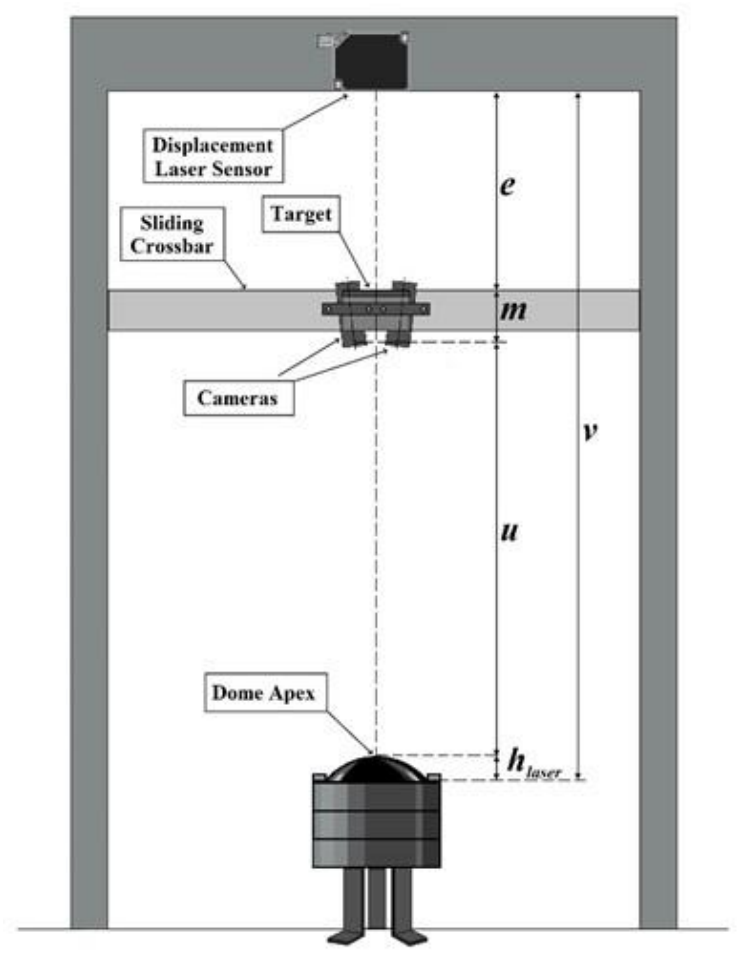

Figure 2. Sketch of the laser sensor measurement (Courtesy of authors [24]).

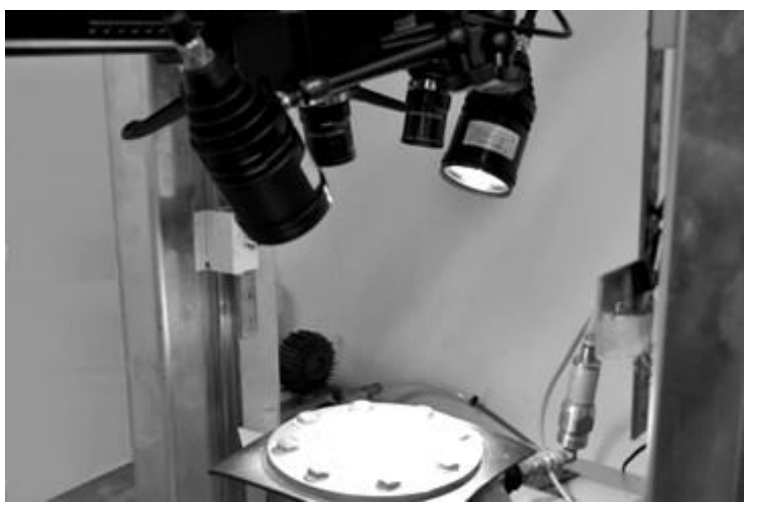

Figure 3. Bulge test setup for 3D-DIC technique (Courtesy of authors [22]). 

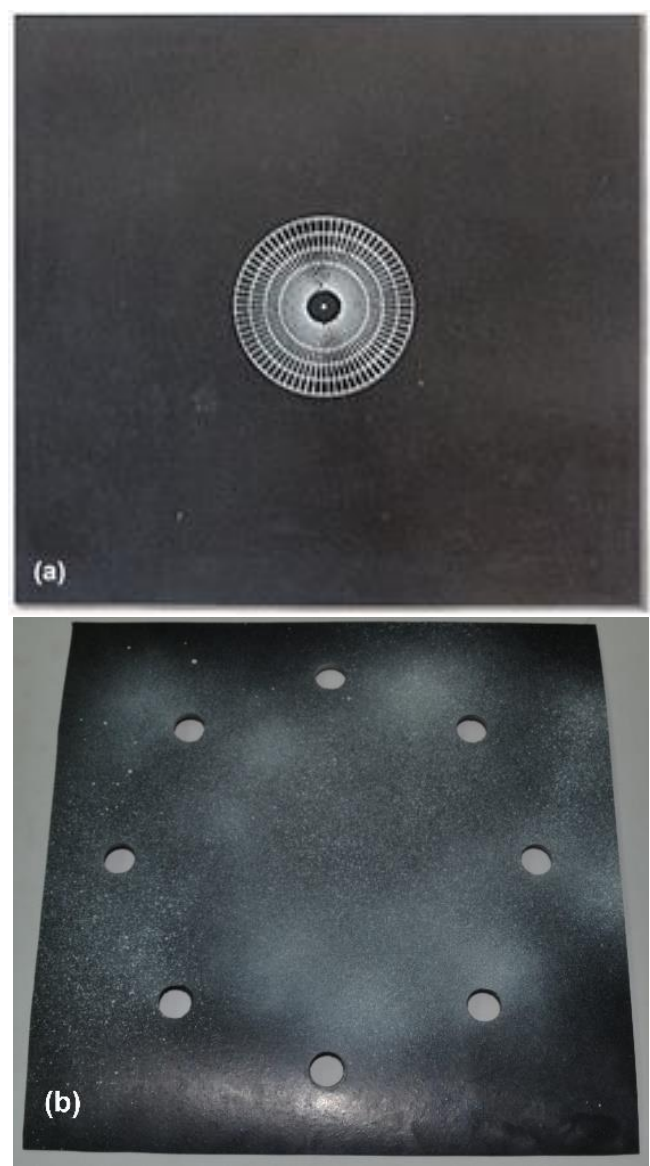

Figure 4. Bulge test sample: (a) side for epipolar reconstruction; (b) side for 3D-DIC reconstruction. In this sample, holes for clamping are already made (Courtesy of authors [22]).

\section{STEREOSCOPIC RECONSTRUCTIONS AND EXPERIMENTAL TESTS}

In the epipolar technique, in order to acquire the whole grid, the cameras separation has not to overcome $10 \%$ of working distance. Thus, adopting a distance of $40 \mathrm{~mm}$ between the two cameras, the distance from the top of the sample (u) was set at $400 \mathrm{~mm}$. Moreover, a focal length of 10,610 pixels, corresponding to $400 \mathrm{~mm}$, and a minimum focusing range from $250 \mathrm{~mm}$ to $\infty$ were set. For accurate displacement measurements, 33 samples at a sample rate of $1 \mathrm{kHz}$ were acquired for each image. Acquisition time required is $1 / 30 \mathrm{~s}$, corresponding to the camera frame rate. For each acquisition, the average of the 33 values, taken as best value, and the standard deviation of the series were computed.

Since the phase shift of a single frame was $1 / 30 \mathrm{~s}$, the resulting synchronization uncertainty could be reasonably estimated in half of it.

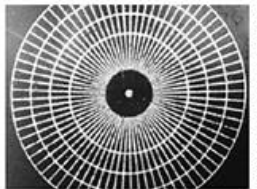

(a)

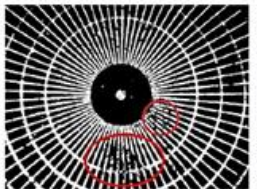

(b)

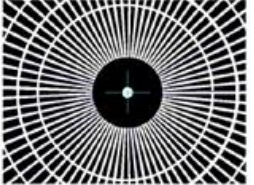

(c)
Figure 5. Image pre-processing stages: (a) grayscale image; (b) convolutionfiltered image; (c) median-filtered image for recovering attenuated-light areas in red encircled of (b) (Courtesy of authors [14]).

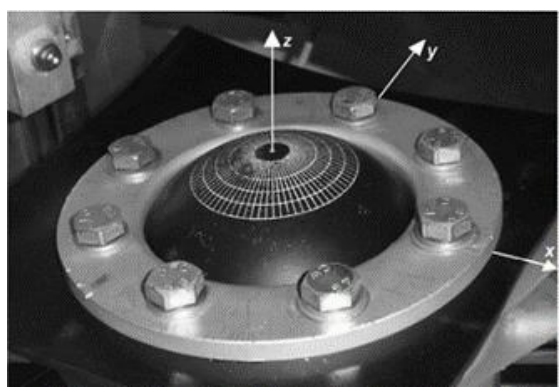

Figure 6. Cartesian reference system (Courtesy of authors [19]).

Due to the slowness of the creep of the specific material tested in the experiments, one acquisition per minute was considered sufficient to capture the relevant information of the phenomenon.

Some pre-processing stages were needed to improve the image sharpness, as in the example shown in Figure 5: the image was first converted to grayscale (Figure $5 \mathrm{a}$ ) and then filtered with the convolution (Figure 5b) and median filters (Figure 5c).

The convolution filter was intended to refine the grid edges by recovering the light intensity lost due to the specimen strain. Next, a smoothing filter (median filter) was applied in order to both reduce the noise introduced by the convolution filter and balance the image. In particular, the size of median filter was the $3 \times 3$ (kernel size) and the type of convolution filter chosen was the "sharpen filter", in order to obtain a more sharpened image allowing a more accurate edge detection.

For the reconstruction, the origin of reference system was set at the centre of the clamping flange, with the vertical $z$-axis passing through the midpoint between the focal centres of cameras, $\mathrm{x}$-axis oriented to the right of the testing machine on the median plane and $\mathrm{y}$-axis perpendicular to $\mathrm{x}$ and $\mathrm{z}$-axes (Figure 6) [19]. The sample was oriented so that the first meridian was along the x-axis. Anticlockwise numeration was chosen for the 73 meridians.

Dome 3D reconstruction was performed using a customized Matlab ${ }^{\circledR}$ algorithm able to fix the markers (Figure 7) [14], corresponding to the intersections between the 73 meridians and the 5 parallels of the screen-printed grid on the specimen. Moreover, this algorithm identifies with red and blue markers the light transitions from dark to light and vice versa, respectively (Figure 7a), selects the homothetic markers (Figure 7b) and compares the distance between two homothetic markers at two consecutive stress states (Figure 7c).

The $3 \mathrm{D}$ reconstruction was implemented also through $3 \mathrm{D}$ DIC. The CCD sensors of the two cameras were of $1624 \times 1236$ pixels, allowing a spatial resolution of $0.092 \mathrm{~mm} /$ pixel. Before acquiring, the optical setup was calibrated with respect to a measure volume equal to $150 \mathrm{~mm} \times 110 \mathrm{~mm} \times 110 \mathrm{~mm}$, taken as reference and vertically set on the centre of the flange (z-axis), in order to ensure that the space gradually occupied by the dome

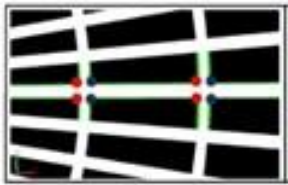

(a)

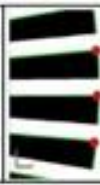

(b)

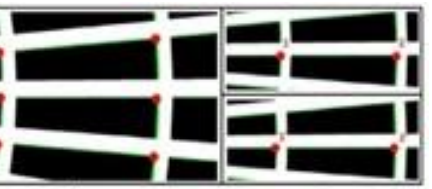

(c)
Figure 7. (a) Light transitions: dark to light (red point) and light to dark (blue point); (b) homothetic markers; (c) comparison of the distance between two homothetic markers at two successive stress states (Courtesy of authors [14]). 


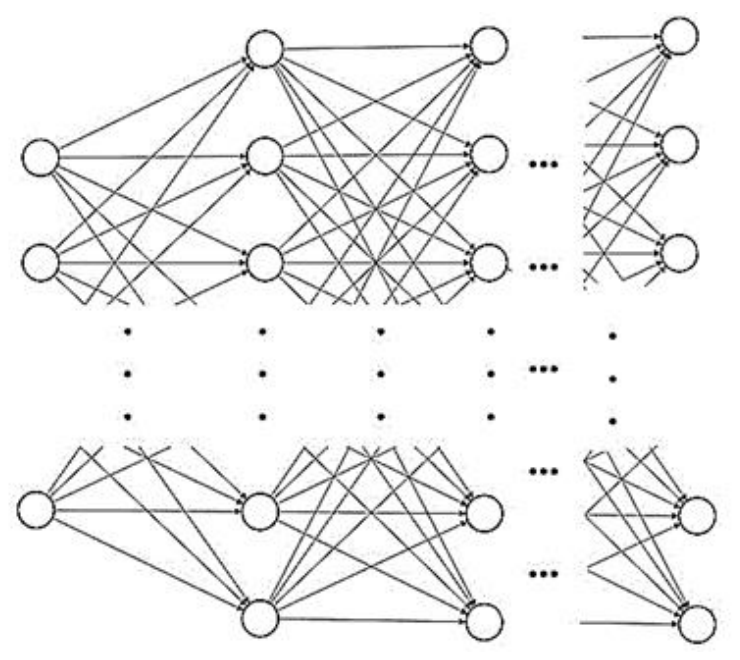

Figure 8. FFNN architecture.

during creep expansion could be enclosed. To do that, a CP20 calibration table $\left(175 \times 140 \mathrm{~mm}^{2}\right)$ was used, while the optics were adjusted on a depth of field equal to $5.6 \mathrm{~mm}$. For post-processing needs, 35 stages in step of $300 \mathrm{~s}$ were acquired during the test. A facets size of $13 \times 13$ pixels with a relative distance of 8 pixels was chosen for computer processing of the DIC meshes.

Each single creep bulge test lasted 180 minutes in order to ensure the complete relaxation of the material within the linear and elastic strains field.

Due to the temperature dependence of the tested elastomer, the temperature was constantly monitored and maintained at $20^{\circ} \mathrm{C} \pm 0.5^{\circ} \mathrm{C}$ for the entire duration of the test.

To use both stereoscopic methods it was necessary to analyse the two sides of each specimen individually. To avoid permanent deformation on the specimen between one test and another, the inflation pressure was kept constant at 0.55 bar that is a value well below the limit pressure. However, the nature of filled hyperelastic materials is such that even specimens from the same sheet can have different mechanical characteristics, especially when analyzed on opposite sides. Therefore, based on the results obtained, it is legitimate to consider that the bias errors may be attributable to any differences in mechanical behavior between one side and the other of the specimen.

\section{NEURAL NETWORK ARCHITECTURE}

The neural network architecture used in this work was a FFNN (Feed Forward Neural Network) composed by 4 layers fully connected [19], [27]. The model had $2 \mathrm{~N}+1$ inputs (the Cartesian coordinates ( $\mathrm{x}, \mathrm{y}$ ) of the $\mathrm{N}$ points resulting from the intersection of meridians with parallels of the 3D image and the inflation pressure value) and $\mathrm{N}$ outputs: the height $\mathrm{z}$ relative of each point.

The structural simplicity of such architecture (Figure 8) allowed achieving a good quality of the outputs without a large waste of computational power and within training period relatively short.

Every layer had the sigmoid activation function and the hidden layers were composed by 30 neurons; only the last layer, the output layer, had a linear activation function.

The model was trained with the dataset built using the Cartesian points $(\mathrm{x}, \mathrm{y}, \mathrm{z})$ from the $3 \mathrm{D}$ reconstruction of the dome and the inflation pressure during creep bulge test, and adopting a Levenberg-Marquardt algorithm as optimizer [28]. MSE (Mean

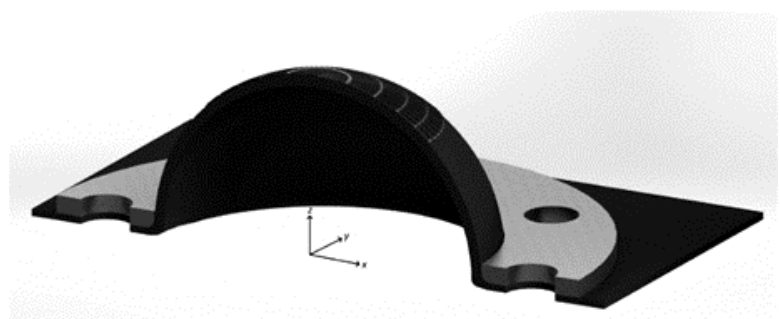

Figure 9. Epipolar geometric reconstruction of the dome (Courtesy of authors [22]).

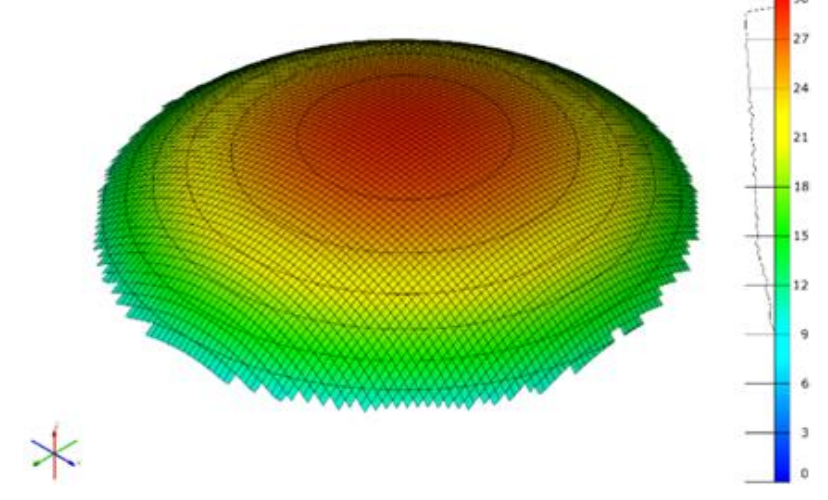

Figure 10. 3D-DIC reconstruction of the dome.

Square Error) loss function and a technique of early stop were used in order to prevent the over-fitting of the model.

\section{ANALYSIS OF THE RESULTS AND CONCLUSIONS}

Figure 9 and Figure 10 show the typical reconstruction of the dome at the end of the test, computed from epipolar geometry and from 3D-DIC algorithm, respectively.

Figure 11a shows the plot of the inflating pressure as a function of the time, highlighting how this parameter, except for the instants in which the pressure regulator acts, remains constant during the whole test, as desired. From the comparison between the trends of the strain as function of the time resulting from the two stereoscopic techniques (Figure 11b), as previously stated, it can be noted that the epipolar reconstruction can exploit a greater number of acquisitions respect the DIC approach (180 vs 35). Therefore, the timing resolution of the epipolar technique is better than the DIC one. However, the spatial resolution is better in the latter, minimizing the error on the measurement of the dome apex height. In effect, while the 3D-DIC spatial resolution is based on a points cloud, the epipolar spatial resolution is based on a limited homothetic markers set.

A way to confirm the better quality of the 3D-DIC reconstruction compared to that obtained with the epipolar geometry is based on the use of FFNN models. Thus, two independent models were obtained by training the FFNN neural network on the acquired datasets. First dataset consists of the acquisitions on the 5 specimens analysed with the epipolar technique, the second one of those acquired on the same specimens with the 3D-DIC technique.

These models have as input the datasets, i.e. the set of the (x, y) coordinates of the acquired points and, as output, the height of the dome apex ( $\mathrm{z}$ ) from each acquisition. The output was used to estimate the bias error with respect to the reference laser value $\left(b_{\text {laser }}\right)$ on the corresponding acquisition. 
Table 1. Comparison between epipolar and DIC FFNN models respect to its own laser measurements. Measurement units in mm.

\begin{tabular}{llllcc}
\hline & $\boldsymbol{h}_{\text {laser (epip. side) }} \pm \boldsymbol{S D}$ & $\boldsymbol{h}_{\text {laser (DIC side) }} \pm \boldsymbol{S D}$ & $\boldsymbol{h}_{\text {epip. }} \pm \boldsymbol{S D}$ & $\boldsymbol{h}_{\text {DIC }} \pm \boldsymbol{S D}$ & Bias $_{\text {epip. }}$ \\
\hline Initial inflation & $27.1 \pm 0.8$ & $25.9 \pm 0.8$ & $28.5 \pm 1.4$ & $26.9 \pm 1.6$ & 1.4 \\
Creep & $29.2 \pm 0.9$ & $28.0 \pm 0.8$ & $30.1 \pm 1.7$ & $28.5 \pm 1.4$ & 0.9 \\
\hline
\end{tabular}
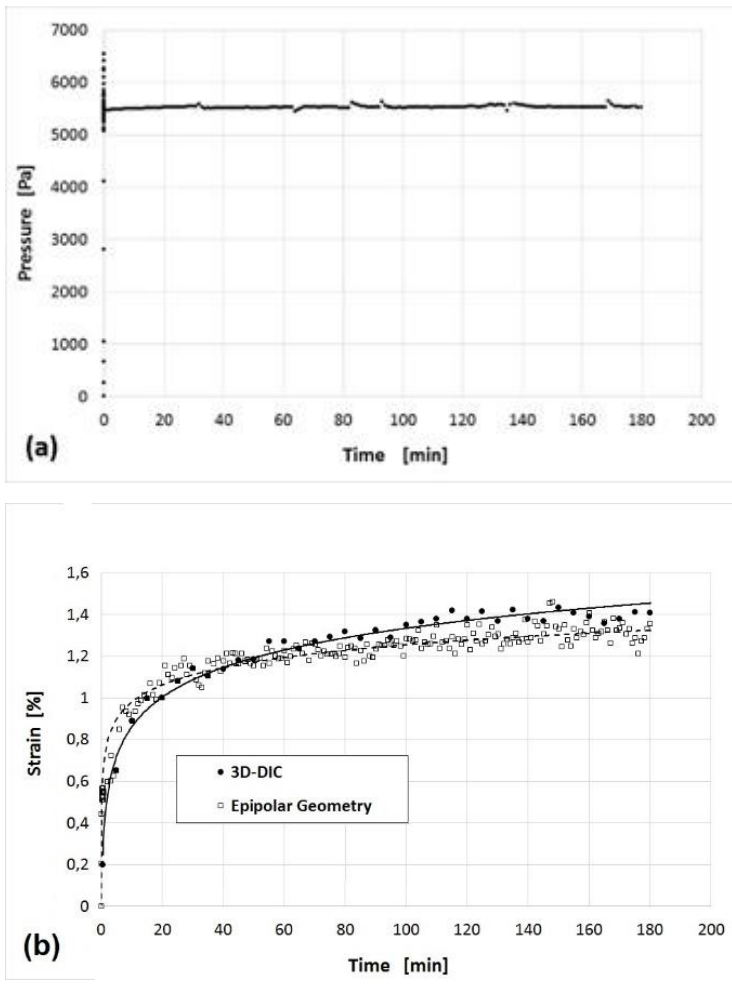

Figure 11. (a) Inflating pressure plot. (b) Trends of the strains from 3D-DIC and epipolar geometry techniques.
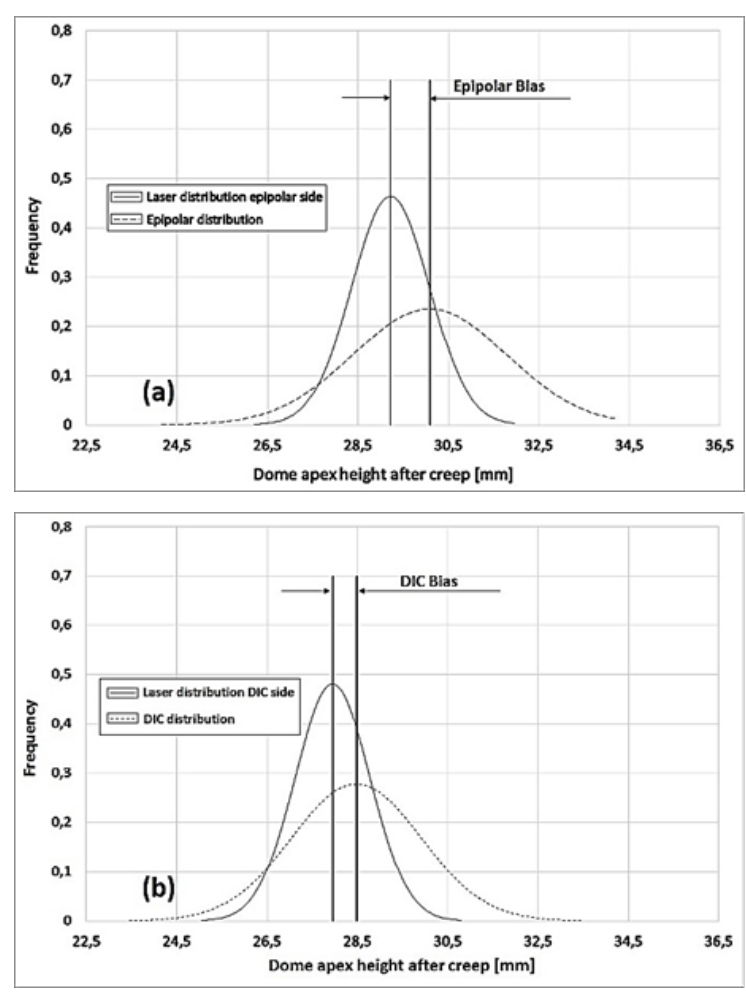

Figure 12. Comparison between Gaussian distributions for the height of the dome apex after creep from (a) laser measurements and epipolar FFNN model and (b) laser measurements and 3D-DIC FFNN model.
Our results showed that the 3D-DIC technique leads to smaller bias errors (Table 1).

With notations of Figure 2, from the direct laser measurement (e), being fixed the working distance (u) at $400 \mathrm{~mm}$ (Section 6), the indirect measurement of the dome apex height (hlaser) is geometrically given by:

$h_{\text {laser }}=v-m-u-e$,

where $m=75 \mathrm{~mm}$ and $v=680 \mathrm{~mm}$.

As previously stated, the measurements provided by the equation (4) were taken as references both for those obtained with the epipolar technique on one side of the specimen, and for those obtained with the 3D-DIC technique on the other side.

Table 1 shows the best values of the dome apex height and the standard deviations $(S D)$ of the measurements from the laser sensor (one for each sample side) and the two FFNN models after creep, where the coverage factor $k$ for the uncertainty of each measurement was considered equal to 1 . Table 1 also shows the bias errors of each of the models with respect to its own reference value. Each bias error is given by the difference, in absolute value, between the FFNN model value and the relative laser value.

Figure 12 plots Gaussian distributions resumed in Table 1. The small deviation observed between the values from FFNN models relative to DIC and epipolar reconstructions, as shown in Figure 12a and Figure 12b is likely due to variations in the hyperelastic behavior of the specimens depending on the side analyzed and not to the inaccuracy of the experimental setup. This is confirmed by the corresponding variation in the reference values provided by the laser sensor.

\section{REFERENCES}

[1] M. Mooney, A Theory of Large Elastic Deformation, J Appl Phys, 11, 1940, 582-592. DOI: $\underline{10.1063 / 1.1712836}$

[2] R. S. Rivlin, Large elastic deformations of isotropic materials IV. Further developments of the general theory, Phil Trans R Soc Lond A, 241, 1948, 379-397. DOI: $10.1098 /$ rsta.1948.0024

[3] L. R. G. Treloar, The Physics of Rubber Elasticity, Clarendon Press, Oxford, 1975.

[4] E. M. Arruda, M. C. Boyce, A three-dimensional constitutive model for the large stretch behavior of rubber elastic materials, J Mech Phys Solids, 41, 1993, 389-412.

DOI: $10.1016 / 0022-5096(93) 90013-6$

[5] O. H. Yeoh, Some Forms of the Strain Energy Function for Rubber, Rubber Chem Technol, 66, 1993, 754-771.

DOI: $\underline{10.5254 / 1.3538343}$

[6] R. W. Ogden, Non-Linear Elastic Deformations, Dover Publications, Mineola, N. Y., 1997, ISBN 978-0-486-69648-5. Z. Chenbing, W. Xinpeng, L. Xiyao, Z. Suoping, W. Haitao, A small buoy for flux measurement in air-sea boundary layer, In Proceedings of the ICEMI 2017. DOI: $10.1109 /$ ICEMI.2017.8265999

[7] J. Y. Sheng, L. Y. Zhang, B. Li, G. F. Wang, X. Q. Feng, Bulge test method for measuring the hyperelastic parameters of soft membranes, Acta Mech 228 (2017) 4187-4197.

DOI: $\underline{10.1007 / \mathrm{s} 00707-017-1945-\mathrm{x}}$ 
[8] M. Koç, E. Billur, O. N. Cora, An experimental study on the comparative assessment of hydraulic bulge test analysis methods, Mater. Des. 32 (2011) 272-281.

DOI: $10.1016 /$ i.matdes.2010.05.057

[9] M. K. Small, W. D. Nix, Analysis of the accuracy of the bulge test in determining the mechanical-properties of thin-films. J Mater Res, 7, 1992, 1553-1563. DOI: $10.1557 /$ JMR.1992.1553

[10] Dimarn, C. Thanadngarn, V. Buakaew, Y. Neamsup, Mechanical properties testing of sheet metal by hydraulic bulge test, Proceedings Vol. 9234, International Conference on Experimental Mechanics 2013 and Twelfth Asian Conference on Experimental Mechanics, 2014. DOI: $10.1117 / 12.2054257$

[11] T. Tsakalakos, The bulge test: a comparison of theory and experiment for isotropic and anisotropic films, Thin Solid Films, 75, 1981, 293-305. DOI: $10.1016 / 0040-6090(81) 90407-7$

[12] J. Diani, M. Brieu, J. M. Vacherand, A. Rezgui, Directional model for isotropic and anisotropic hyperelastic rubber-like materials, Mech Mater, 36, 2004, 313-321.

DOI: 10.1016/S0167-6636(03)00025-5

[13] M. Sasso, G. Palmieri, G. Chiappini, D. Amodio, Characterization of hyperelastic rubber-like materials by biaxial and uniaxial stretching tests based on optical methods, Polym Test, 27, 2008, 995-1004.

DOI: $10.1016 /$ i.polymertesting. 2008.09.001

[14] M. Calì, F. Lo Savio, Accurate 3D reconstruction of a rubber membrane inflated during a Bulge Test to evaluate anisotropy, Advances on Mechanics, Design Engineering and Manufacturing - Lecture Notes in Mechanical Engineering, Springer, 2017, 12211231.

DOI: $10.1007 / 978-3-319-45781-9 \quad 122$

[15] M. Sigvant, K. Mattiasson, H. Vegter, P. Thilderkvist, A viscous pressure bulge test for the determination of a plastic hardening curve and equibiaxial material data, Int J Mater Form, 2009, 2, 235242.

DOI: $10.1007 / \mathrm{s} 12289-009-0407-\mathrm{V}$

[16] L. Vitu, N. Laforge, P. Malécot, N. Boudeau, S. Manov, M. Milesi, Characterization of zinc alloy by sheet bulging test with analytical models and digital image correlation, AIP Conference Proceedings, 2018, Proceedings of the 21st International Esaform Conference on Material Forming, Palermo, Italy. DOI: $10.1063 / 1.5035022$

[17] C. Feichter, Z. Major, R. W. Lang, Methods for Measuring Biaxial Deformation on Rubber and Polypropylene Specimens, in: Gdoutos E. E. (eds), Experimental Analysis of Nano and Engineering Materials and Structures, 2007, 273-274. DOI: $10.1007 / 978-1-4020-6239-1 \quad 135$

[18] J. Neggers, J. P. M. Hoefnagels, F. Hild, S. Roux, M. G. D. Geers, Global digital image correlation for pressure-deflected membranes. In G. A. Shaw, B. C. Prorok, \& L-VA. Starman
(Eds.), Proceedings of the 2012 Annual Conference on Experimental and Applied Mechanics, 6, 2012, 135-140. New York: Springer.

DOI: $10.1007 / 978-1-4614-4436-7 \quad 20$

[19] F. Lo Savio, G. Capizzi, G. La Rosa, G. Lo Sciuto, Creep assessment in hyperelastic material by a 3D Neural Network Reconstructor using bulge testing. Polymer Testing 63 (2017) 6572 .

DOI: $10.1016 /$ i.polymertesting. 2017.08.009

[20] T. Moons, L. Van Gool, M. Vergauwen, 3D Reconstruction from Multiple Images, Part 1: Principles, Foundations and Trends ${ }^{\circledR}$ in Computer Graphics and Vision, Vol. 4, No. 4 (2008) 287-398. DOI: $10.1561 / 0600000007$

[21] J. Li, Z. Miao, X. Liu, Y. Wan, 3D Reconstruction based on stereovision and texture mapping, in Paparoditis N., PierrotDeseilligny M., Mallet C., Tournaire O. (Eds), IAPRS, Vol. XXXVIII, Part 3B - Saint-Mandé, France, 2010.

[22] D. Alizzio, F. Lo Savio, M. Bonfanti, G. Garozzo, A new approach based on neural network for a $3 \mathrm{~d}$ reconstruction of the dome of a bulge tested specimen. CEUR Workshop Proceedings ICYRIME 2020, 2768 (2020), pp. 54-58. Online [Accessed 22 December 2021] http://hdl.handle.net/20.500.11769/496378.

[23] Z. Zhang, A Flexible New Technique for Camera Calibration, IEEE T Pattern Anal, 22, 2000, pp. 1330-1334. DOI: $10.1109 / 34.888718$

[24] F. Lo Savio, G. La Rosa, M. Bonfanti, A new theoreticalexperimental model deriving from the contactless measurement of the thickness of bulge-tested elastomeric samples. Polymer Testing 87 (2020), 106548 DOI: $10.1016 /$ i.polymertesting. 2020.106548

[25] F. Lo Savio, M. Bonfanti, G. M. Grasso, D. Alizzio, An experimental apparatus to evaluate the non-linearity of the acoustoelastic effect in rubber-like materials, Polymer Testing 80 (2019), 106133. DOI: $10.1016 /$ i.polymertesting. 2019.106133

[26] F. Lo Savio, M. Bonfanti, A novel device for measuring the ultrasonic wave velocity and the thickness of hyperelastic materials under quasi-static deformations, Polymer Testing 74 (2019), pp. 235-244.

DOI: $10.1016 /$ i.polymertesting.2019.01.005

[27] L. W. Peng, S. M. Shamsuddin, 3D Object Reconstruction and Representation Using Neural Networks, Proceedings of the 2nd International Conference on Computer Graphics and Interactive Techniques in Australasia and Southeast Asia 2004, Singapore, pp. 139-147. DOI: $10.1145 / 988834.988859$

[28] J. J. Moré, The Levenberg-Marquardt algorithm: Implementation and theory, Numerical Analysis, LNM 630 (2006), pp. 105-116. DOI: $\underline{10.1007 / \mathrm{BFb} 0067700}$ 